\title{
The Population Pharmacokinetics of Rifampicin in Japanese Pulmonary Tuberculosis Patients
}

\author{
Authors \\ Takahiro Nishimura1, Haruichi Kohno², Hideaki Nagai³, Daisuke Maruoka4, Yuichi Koike², Masahiro Kobayashi ${ }^{5}$, \\ Koichiro Atsuda 5
}

\section{Affiliations}

1 Department of Pharmacy, National Cancer Center Hospital East, Japan

2 School of Pharmaceutical Sciences, Ohu University, Japan

3 National Hospital Organization, Tokyo National Hospital, Japan

4 Department of Pharmacy, National Hospital Organization, Tokyo National Hospital, Japan

5 School of Pharmaceutical Sciences, Kitasato University, Japan

\author{
Key words \\ population pharmacokinetics, rifampicin, Japanese \\ tuberculosis patients
}

received 26.11.2019

accepted $\quad 14.02 .2020$

Bibliography

DOI https://doi.org/10.1055/a-1122-8129

Published online: 19.3 .2020

Drug Res 2020; 70: 199-205

(c) Georg Thieme Verlag KG Stuttgart · New York

ISSN 2194-9379

Correspondence:

Takahiro Nishimura MS

Department of Pharmacy, National Cancer Center Hospital East 6-5-1, Kashiwanoha

Kashiwa-shi

277-8577 Chiba

Japan

Tel.: + 81-4-7133-1111, Fax: + 81-4-7134-6879

takahiro411130@hotmail.com

\begin{abstract}
In Japan, tuberculosis has been recognized as one of the major infections requiring urgent measures because of its high morbidity rate even now especially in elderly people suffering from tuberculosis during the past epidemic and its reactivation. Hence, many Japanese clinicians have made efforts to suppress the onset of tuberculosis and treat it effectively. The objectives of this study are to (1) identify covariate(s) that may explain the variation of rifampicin, which is the key antitubercular agent, under the steady-state by evaluating its population pharmacokinetics and (2) to propose an appropriate dosing method of rifampicin to Japanese patients. For this purpose, serum concentration-time data were obtained from $138 \mathrm{pa}-$ tients receiving rifampicin (300-450 mg) and isoniazid (300$400 \mathrm{mg}$ ) every day over 14 days, and analyzed using nonlinear mixed effects model. Thereby, population pharmacokinetic parameters were estimated followed by elucidating relations between the parameters and statistical factors. The analysis adopted one-compartment model including Lag-time by assuming that the absorption process is $0+1$ st order. The analyses demonstrate that meal affected the bioavailability, primary absorption rate constant, and zero order absorption time in the constructed model. A body weight calculated from the power model was selected as the covariate by the Stepwise Covariate Model method and found to highly affect the clearance in the range from $-31.6 \%$ to $47.4 \%$. We conclude that the dose in Japanese tuberculous patients can be well estimated by the power model formula and should be taken into consideration when rifampicin is administered.
\end{abstract}

\section{Introduction}

In Japan, 15000 or more people newly developed tuberculosis (TB) including elderly people suffering from its prevalence in the past and reactivation, and 2000 or more died in a year [1]. The incidence rate of TB in 2018 is 12.3 for the 100000 population in Japan, down 0.6 points from the previous year [2]. The rate is low compared to that in neighboring Asian countries, but still higher than that in de- veloped countries (UK: 7.1, France: 7.4, Canada: 4.8, USA: 2.6) [3]. Thus, Japan is now categorized to a medium prevalence country for TB in spite of its high hygienic status.

The basic goal of current TB medicine is to eradicate tuberculosis organisms living in TB patients. For this purpose, the following standard tuberculosis chemotherapy (STC) has been proposed and 
carried out in Japan [4]. In STC, first, the first-line drugs (designated as a) consisting of rifampicin (RFP) + isoniazid (INH) + pyrazinamide (PZA) are used in combination with the first-line drug (designated as b) consisting of either ethanbutol (EB) or streptomycin $(\mathrm{SM})$. At 2 months later from the initiation of the treatment, patients are further treated with RFP + INH for 4 months. This 6-month therapy is the strongest and shortest one that can achieve the therapeutic goal of "eradication of bacteria". Thereafter, treatment with RFP + INH + EB (or SM) for 3 months followed by RFP + INH for 7 months is carried out to ensure the "eradication". As described above, RFP has occupied the center position of STC; this is the reason for us to focus on RFP in this study, although RFP is a potent inducer of some types of cytochrome P450s (CYPs) and affects the metabolism of other combined drugs so called drug-drug interaction [5]. Furthermore, constantly keeping serum concentration of RFP at its effective levels is clinically important in each patient but relatively difficult because of large inter-individual variation of its bioavailability (F1) due to the genetic polymorphism of the p-glycoprotein and organic anion transport polypeptide 1B1 (SLCO1B1 rs4149032), the drug-drug interactions, etc [6-8].

To provide useful information for improvement of TB chemotherapy, the authors conducted a population-pharmacokinetics (PPK) analysis of RFP in Japanese TB patients followed by elucidating the factors which may influence the effective serum concentration of RFP.

\section{Patients and Methods}

This study was approved by the Ethical Review Board of National Hospital Organization of Tokyo National Hospital. The subjects were 138 Japanese TB patients who admitted to the hospital from April 2005 to March 2010 and were administered RFP (300-450 mg) before or after meal and INH (300-400 mg) after meal over 14 days or more. Blood was collected at the request of doctors to monitor the blood concentration of RFP. The serum concentration and the biochemical data were retrospectively collected from medical or electronic medical records. The patient physiological factors that may affect RFP pharmacokinetics were assumed to be age, body weight (WGT), gender, ALT (alanine transaminase), AST (aspartate transaminase), BUN (blood urea nitrogen), үGTP (gamma-glutamyl transpeptidase), serum creatinine, total bilirubin, and eGFR (estimated glomerular filtration rate) and $>$ Table 1 shows their background information. The eGFR was determined using the calculation method defined by the Japanese Society of Nephrology [9]. The equations to determine eGFR are as follows: eGFRcreat $(\mathrm{mL} /$ $\left.\min / 1.73 \mathrm{~m}^{2}\right)=194 \times \mathrm{Cr}^{-1.094} \times$ age $^{-0.287}$ for males and eGFRcreat
$\left(\mathrm{mL} / \mathrm{min} / 1.73 \mathrm{~m}^{2}\right)=194 \times \mathrm{Cr}^{-1.094} \times$ age $^{-0.287} \times 0.739$ for females The time of blood collection was set at one point each during an absorption phase (1-3 h after RFP administration), elimination phase ( 4 to $8 \mathrm{~h}$ after RFP administration), and at trough. The serum concentration was determined by the method of Kubo et al [10]. Briefly, in the quantitative analysis of RFP in serum, serum was deproteinized by adding 5 volumes of methanol followed by centrifugation. The concentration of RFP in the sample thus obtained was determined with HPLC equipped with a C18 reverse phase column under the following conditions: mobile phase; $38 \%$ acetonitrile solution containing $0.01 \mathrm{M}$ sodium acetate $(\mathrm{pH} 7.0)$, flow rate of mobile phase; $1.5 \mathrm{~mL} / \mathrm{min}$, and detection; UV $340 \mathrm{~nm}$. The calibration curve was linear up $20 \mu \mathrm{g} / \mathrm{mL}$ of RFP. At the concentration of $8.0 \mu \mathrm{g} /$ $\mathrm{mL}$, intraday and interday variations were small and $1.0 \%(<1>n<1$ $I>=10)$ and $1.5 \%(<|>n<| I>=10)$, respectively.

For PPK analysis of RFP, NONMEM (Nonlinear Mixed Effects Model) program Version VII ${ }^{\mathrm{TM}}$ was used. The NONMEM algorithm used was first order conditional estimation with interaction. The basic model was selected from the following three models: the pharmacokinetic model, the measurement error model, and the mixed effect model. The pharmacokinetic model of RFP was analyzed using a 1-compartment model with a lag-time of absorption (ALAG1) including the zero-order absorption (R1) followed by the first order absorption (Ka) as shown in > Fig. 1. The exponential error model shown below was used as the mixed effect model that displays inter-individual variation of pharmacokinetic parameters.

$\mathrm{Pi}=\mathrm{P} \times \exp (\eta \mathrm{i})$

- Table 1 Patient background data of physiological factors affecting RIF pharmacokinetics.

\begin{tabular}{|l|l|l|}
\hline & Number & Median [min - max] \\
\hline Gender (male/female) & $110 / 28$ & \\
\hline Age (year) & & $58[21-90]$ \\
\hline WGT $(\mathrm{kg})$ & & $51.5[32.0-58.0]$ \\
\hline ALT $(\mathrm{U} / \mathrm{L})$ & & $14.0[6.0-285]$ \\
\hline AST $(\mathrm{U} / \mathrm{L})$ & & $21.0[11.0-126]$ \\
\hline BUN $(\mathrm{mg} / \mathrm{dL})$ & & $11.7[3.7-55.5]$ \\
\hline Gamma GTP $(\mathrm{U} / \mathrm{L})$ & & $44[10-335]$ \\
\hline Serum Creatinine $(\mathrm{mg} / \mathrm{dL})$ & & $0.68[0.25-3.72]$ \\
\hline Total Bilirubin $(\mathrm{mg} / \mathrm{dL})$ & & $0.36[0.18-3.96]$ \\
\hline eGFR $\left(\mathrm{mL} / \mathrm{min} / 1.73 \mathrm{~m}^{2}\right)$ & & $90.4[14.3-283]$ \\
\hline
\end{tabular}

ALT: Alanine aminotransferase, AST: Aspartate aminotransferase, BUN: Blood urea nitrogen, eGFR: Estimated glomerular filtration rate, GTP: Glutamyl transpeptidase, RIF: Rifampin, WGT: Body weight.

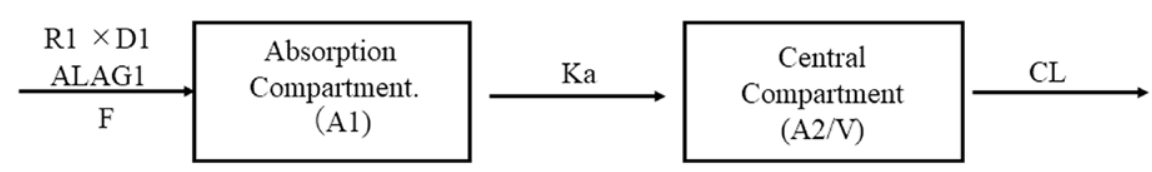

-Fig. 1 Structure model. R1: Rate of zero order input, D1: Duration (if fasted condition, D1=0), ALAGl: Lag time of absorption (start of drug input), F: Bioavailability (if fed condition, $F=1$ ), Ka: 1st order absorption constant, A1: Drug amount in absorption compartment, A2: Drug amount in central compartment, V: Distribution volume, CL: Systemic clearance. 
Where, $\mathrm{P}$ is a mean value of pharmacokinetic parameters, $\mathrm{Pi}$ is a pharmacokinetic parameter value of subject $i$, and $\eta i$ is an inter-individual variation, which is a difference between the mean value $(\mathrm{P})$ and a patient intrinsic value $(\mathrm{Pi})$. The $\eta$ is to follow a normal distribution with a mean value 0 and variance $\omega^{2}$. Correlation is to be permissible for the inter-individual variation of $C L t / F$ and $V / F$. The exponential error model shown below was used for the intra-individual variation model.

$\mathrm{Cpij}=\mathrm{Cij} \times \exp (\varepsilon)$

Where, $\mathrm{Cij}$ is the measured drug concentration in serum at time $\mathrm{j}$ of subject i, Cpij is the estimated drug concentration in serum at time $j$ of subject $i$, and $\varepsilon$ displays residual variation and is to follow normal distribution with mean value 0 and variance $\sigma^{2}$.

The absorption rate constant and bioavailability, which are likely parameters pharmacokinetically affected by diet, were examined. However, since these parameters are not clearly relevant from a pharmacokinetic point of view, they were not included as the candidate of covariates.

After constructing the base model, we constructed a model according to the following steps.

For the construction of absorption model, in addition to the primary absorption, the zero-order absorption, the primary absorption with lag time, $0+1$ order absorption, and $0+1$ order absorption with lag time were examined. Among them, the model giving the lowest OFV was that with the $0+1$ first-order absorption including the lag time.

We examined the effect of diet on bioavailability, duration of zero-order absorption, lag time, and first-order absorption rate constant. The results showed that the diet had a significant effect on any of the parameters. We therefore select dietary effects to incorporate them into the duration and bioavailability of zero-order absorption by which the lowest OFV was obtained for all. The authors therefore chose to incorporate dietary effects into the duration and bioavailability of zero-order absorption with the lowest OFV for all the parameters.

According to the procedure described above, a model to which the absorption and dietary effects were incorporated was created from the base model as the structural model. The effects of diet on the parameters, absorption rate constant and bioavailability, which are thought to affect the pharmacokinetics, were examined, but those parameters were not clearly related to the diet from a pharmacokinetic view point and not included as a covariate candidate.

In exploratory search of covariates, Stepwise Covariate Model (SCM) method was adopted. In the Forwarding Step of the SCM method, a $X$-square test was performed with $p<0.01$, and statistically significant covariates were selected. In the Backwarding Step, significant covariates were selected with $p<0.001$, and the final model was constructed by using the covariates remaining as the result as the factors that affect the pharmacokinetics of RFP. In the incorporation of covariates, continuous quantities were incorpo- rated by linear (Eq. 1) and power (Eq. 2), and discrete quantities were incorporated based on Eq. 3.

$$
\begin{aligned}
& \theta_{i}=\left(1+\theta_{\text {cov }} \times\left(\operatorname{cov}_{i}-\text { median }(\operatorname{cov})\right)\right) \\
& \theta_{i}=\left(\frac{\operatorname{cov}_{i}}{\operatorname{median}(\operatorname{cov})}\right)^{\theta_{\operatorname{cov}}} \\
& \text { If }(\operatorname{cov}=0) \theta_{i}=1 \\
& \text { If }(\operatorname{cov}=1) \theta_{i}=1+\theta_{\text {cov }}
\end{aligned}
$$

The authors confirmed the validity of the results based on goodness of fit and visual predictive check (VPC). The VPC was simulated 1000 times and performed by comparing the $95 \%$ prediction interval of the estimated serum concentration with the actual values at each time point. The validity of the obtained parameters was examined by the bootstrap method in which the analysis was conducted by performing restoration extraction 1000 times from the data set using random numbers. The authors checked the validity of the determined parameters by comparing the $95 \%$ confidence intervals of the analysis results obtained with the confidence intervals calculated from the parameters and their standard errors in the final model.

\section{Results}

RFP (300-450 mg) was given before or after meals to 138 Japanese TB patients and the blood samples were obtained at 662 points to elucidate the effects of covariates on pharmacokinetics of RFP.

- Table 1 shows the background data of physiological factors which may affect RFP pharmacokinetics.

- Table 2 represents the population pharmacokinetic parameters calculated from the basic model.

Since the objective function value (OFV) became the lowest when WGT was incorporated into $\mathrm{CL}$ in a linear model in the Forwarding step, this covariate was adopted in the final model. In addition, since the incorporation of WGT into $\mathrm{CL}$ in the power model further reduced the OFV, WGT was selected as the covariate. There was no other model in which the OFV decreased with statistical significance at $\mathrm{p}<0.01$. In the Backwarding step, WGT incorporated by the power model was adopted as the covariate, because OFV increased by the incorporation of WGT into the $\mathrm{CL}$ in the linear model $(p<0.001)$. As a result, the final model was constructed by using equations of each pharmacokinetic parameter shown in > Fig. 2.

- Figure 3 left shows the goodness of fit for the predicted values of population average (population predictions: PRED) calculated from the population pharmacokinetic parameters of the final model against the measured values of serum concentration of RFP (Cp, Observations) in the individual patients. - Figure 3 right represents the goodness of fit for the individual predicted values (individual predictions: IPRED) based on the individual patient parameters determined by Bayesian estimation against Observations. This result confirms that the final model well reflects the serum concentration-time profile of RFP. 
We examined the predictability over time for the predicted values estimated from the model by VPC and Observations (- Fig. 4). As a result, the medians and $95 \%$ tile intervals (upper and lower limits) of the measured values almost overlap the medians and $95 \%$ tile prediction intervals of the serum concentration determined by the model; thus, the predictability of this final model is shown to be good.

The accuracy of parameter estimation was examined using the bootstrap method ( $\triangleright$ Table 3 ). The median values of the popula-

- Table 2 Population parameter estimates.

\begin{tabular}{|l|c|l|}
\hline Parameters & Basic model & Final model \\
\hline Objective function value & 916.308 & 871.120 \\
\hline CL/F (L/hr) & 14.4 & $15.2(3.7 \%)$ \\
\hline WGT on CL/F & - & $0.978(20.4 \%)$ \\
\hline V/F (L) & 5.57 & $2.09(36.2 \%)$ \\
\hline $\begin{array}{l}\text { Relative bioavailability } \\
\text { without food }\end{array}$ & 1.43 & $1.32(6.8 \%)$ \\
\hline $\begin{array}{l}\text { Relative bioavailability } \\
\text { with food }\end{array}$ & 1.00 & 1.00 \\
\hline Ka without food (1/hr) & 0.260 & $0.248(3.9 \%)$ \\
\hline Ka with food (1/hr) & 0.317 & 0.300 \\
\hline Duration without food (hr) & 1.84 & $1.58(11.6 \%)$ \\
\hline Duration with food (hr) & 0 & 0 \\
\hline Lag time (hr) & 1.21 & $1.43(3.0 \%)$ \\
\hline BSV on CL/F & 0.0908 & $0.152(14.9 \%)$ \\
\hline BSV on V/F & 0.236 & $2.30(38.6 \%)$ \\
\hline \begin{tabular}{l} 
BSV on bioavailability \\
\hline BSV on Ka
\end{tabular} & 0.0935 & $0.00000398(49.5 \%)$ \\
\hline BSV on duration with food & 0.0661 & $0.0624(36.9 \%)$ \\
\hline BSV on lag time & 0.702 & $0.612(38.6 \%)$ \\
\hline $\begin{array}{l}\text { Proportional component } \\
\text { of residual error }\end{array}$ & 0.0486 & $0.0168(41.0 \%)$ \\
\hline $\begin{array}{l}\text { BSV: Between subject variability, CL: Systemic clearance of rifampin, } \\
\text { F: Relative bioavailability to that in fasted conditions (F } 1 \text { in fed } \\
\text { conditions), Ka: 1st order absorption constant, V: Volume of distribu- } \\
\text { tion, WGT: body weight, () : RSE\% of parameter estimate. }\end{array}$ \\
\hline
\end{tabular}

tion pharmacokinetic parameters determined by the final model and those determined by the bootstrap method were almost the same. Moreover, the $95 \%$ confidence intervals obtained from the population pharmacokinetic parameters of the final model and the standard errors of parameter estimation were approximately in the same range of those obtained from the bootstrap method. The results described above confirm that this model can calculate the parameters stably with sufficient estimation accuracy.

\section{Discussion}

This population pharmacokinetic analysis model provided good result in the goodness of fit. In the VPC, the median and the $95 \%$ pre-

$$
\begin{aligned}
& C L_{i}=15.2 \times\left(\frac{\mathrm{WGT}}{51.5}\right)^{0.978} \times e^{\eta_{C L, i}} \\
& V_{i}=2.09 \times e^{\eta_{V, i}} \\
& F_{i}= \begin{cases}1.00 \times e^{\eta_{F, i}} & \text { (Fed) } \\
1.32 \times e^{\eta_{F, i}} & \text { (Fast) }\end{cases}
\end{aligned}
$$

$$
\begin{aligned}
& K a_{i}= \begin{cases}0.248 \times e^{\eta_{K a, i}} & (\text { Fed }) \\
0.248 \times 1.21 \times e^{\eta_{K a, i}} & \text { (Fasted) }\end{cases} \\
& \text { Duration }_{i}= \begin{cases}1.58 \times e^{\eta_{D l, i}} & \text { (Fed) } \\
0 & \text { (Fasted) }\end{cases}
\end{aligned}
$$

$$
\operatorname{LagTime}_{i}=1.43 \times e^{\eta_{A L A G, i}}
$$

- Fig. 2 Equation of each pharmacokinetic parameter in the final model. CL: Systemic clearance of rifampin, V: Distribution volume, F: Bioavailability of absorption (if fed condition, $\mathrm{F}=1$ ), Ka: 1st order absorption constant, D1: Duration (if fasted condition, D1=0), ALAGI: Lag time of absorption (start of drug input), i: individual

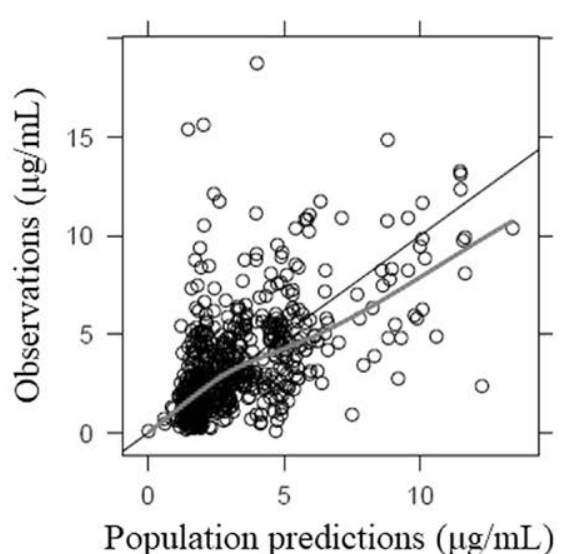

Population predictions $(\mu \mathrm{g} / \mathrm{mL})$

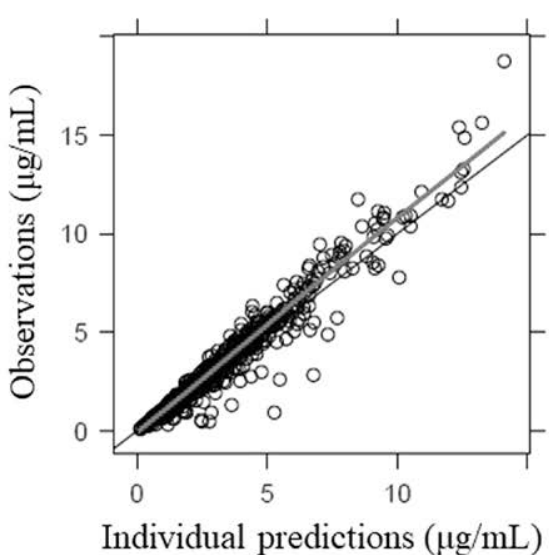

Individual predictions $(\mu \mathrm{g} / \mathrm{mL})$

Fig. 3 Goodness of fit. 
- Table 3 Model validation: Bootstrap method.

\begin{tabular}{|c|c|c|c|c|c|c|c|c|}
\hline & $\mathrm{CL}(\mathrm{L} / \mathrm{hr})$ & $\mathrm{V}(\mathrm{L})$ & Ka (1/hr) & $\begin{array}{l}\text { Duration } \\
\text { (hr) }\end{array}$ & $\begin{array}{l}\text { Lag time } \\
\text { (hr) }\end{array}$ & FOOD on $F$ & FOOD on Ka & $\begin{array}{l}\text { CL/F on } \\
\text { WGT }\end{array}$ \\
\hline \multicolumn{9}{|c|}{ Final Parameter Estimate } \\
\hline means & 15.2 & 2.09 & 0.248 & 1.58 & 1.43 & 1.32 & 1.21 & 0.978 \\
\hline $2.50 \%$ & 14.1 & 0.605 & 0.229 & 1.22 & 1.35 & 1.15 & 1.05 & 0.585 \\
\hline $97.50 \%$ & 16.3 & 3.57 & 0.267 & 1.94 & 1.52 & 1.50 & 1.37 & 1.37 \\
\hline \multicolumn{9}{|c|}{ Bootstrap results } \\
\hline medians & 15.2 & 1.95 & 0.248 & 1.58 & 1.45 & 1.33 & 1.22 & 0.985 \\
\hline $2.50 \%$ & 14.0 & 0.700 & 0.226 & 1.24 & 1.34 & 1.17 & 1.07 & 0.575 \\
\hline \multirow[t]{2}{*}{$97.50 \%$} & 16.3 & 3.55 & 0.270 & 1.93 & 1.56 & 1.49 & 1.38 & 1.40 \\
\hline & BSV CL/F & BSV V/F & BSV Ka & $\begin{array}{l}\text { BSV } \\
\text { Duration }\end{array}$ & $\begin{array}{l}\text { BSV Lag } \\
\text { time }\end{array}$ & BSV F1 & $\begin{array}{l}\text { residual } \\
\text { variability (exp) }\end{array}$ & \\
\hline \multicolumn{9}{|c|}{ Final Parameter Estimate } \\
\hline means & 0.152 & 2.30 & 0.0624 & 0.612 & 0.0168 & $3.98 \mathrm{E}-06$ & 0.0495 & \\
\hline $2.50 \%$ & 0.108 & 0.565 & 0.0173 & 0.427 & 0.00330 & $1.28 \mathrm{E}-07$ & 0.0320 & \\
\hline $97.50 \%$ & 0.196 & 4.04 & 0.107 & 0.797 & 0.0303 & $7.84 \mathrm{E}-06$ & 0.0670 & \\
\hline \multicolumn{9}{|c|}{ Bootstrap results } \\
\hline medians & 0.149 & 2.50 & 0.0629 & 0.609 & 0.0159 & $3.99 \mathrm{E}-06$ & 0.0485 & \\
\hline $2.50 \%$ & 0.105 & 1.04 & 0.0208 & 0.419 & 0.00648 & 3.09E-08 & 0.0328 & \\
\hline $97.50 \%$ & 0.193 & 5.42 & 0.115 & 0.868 & 0.0281 & $1.75 \mathrm{E}-03$ & 0.0663 & \\
\hline
\end{tabular}

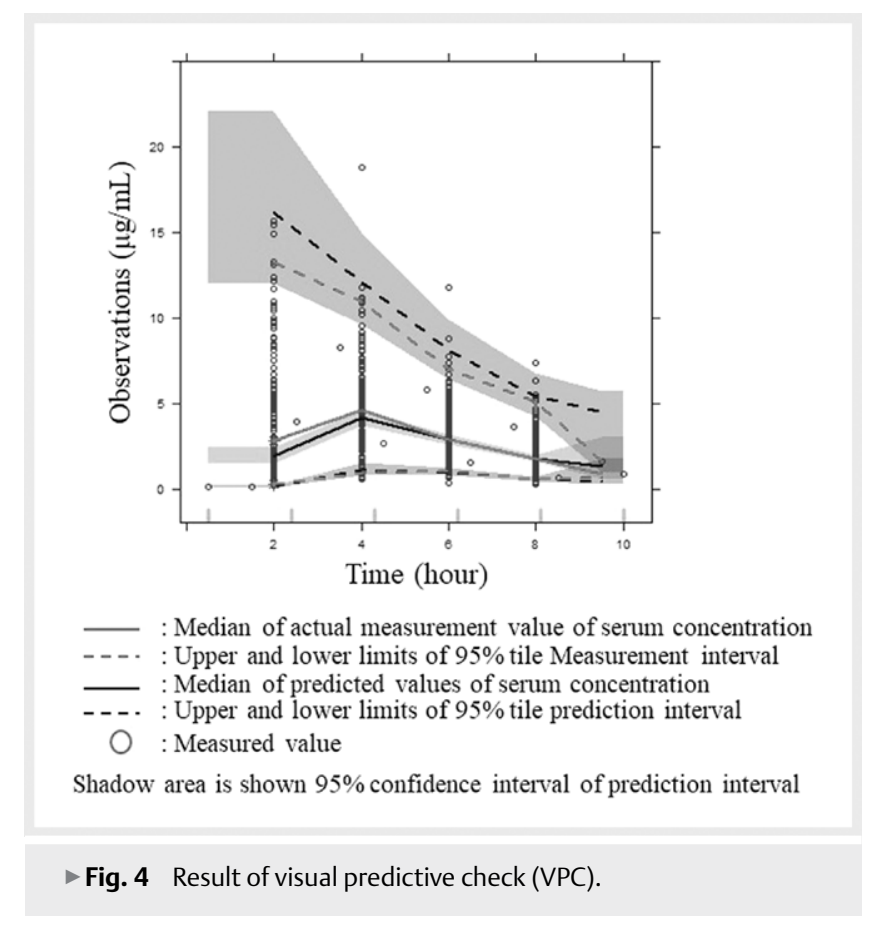

diction interval well reflected the measured values. The parameters and their confidence intervals obtained from the final model were almost the same as those obtained by bootstrap. Based on these observations, we have concluded that the models and PPK parameters having sufficient precision provided the present good analysis results; proving the validity of the analysis.
The model containing the absorption process of RFP with zeroorder followed by the first-order absorption was used in the base model. The solubility of RFP is reported to be high under the acidic conditions in the stomach, but low from the small intestine due to its high $\mathrm{pH}$, while the absorbability from the small intestine is high [11]. Thus, the low solubility of RFP in the small intestine may cause to limit its absorption from this tissue site. In the models used in this study, better fitting was obtained by the model in which the dissolution rate was limited by the zero-order process, followed by the first-order process for the absorption of RFP into the body. Therefore, we have thought that this model well represents the physicochemical and biological properties of RFP.

A model in which the bioavailability and the absorption rate constant in the digestive tract fluctuate due to the influence of meals was used in this study; this also indicating that the variations of the $\mathrm{pH}$ and the content of water serving as the solvent in the digestive tract may affect the absorption of RFP. Therefore, the model probably reflected the physicochemical properties of RFP.

The WGT was selected as the covariate affecting CL by the SCM method. The effect of WGT on CL is relatively large ranging from $-31.6 \%$ to $47.4 \%$, which was able to be calculated from the power model of $>$ Fig. 2 . This suggests that the exposure can vary with the WGT; indicating that exposure will increase in patients with light WGT. Since RFP is, however, a drug with a relatively wide safety margin, it is not considered necessary to adjust the exposure by a dose adjustment. Similarly, although the exposure was predicted to increase up to approximately $32 \%$ in the F1 under the conditions before meal, the administration of RFP for which the effect of diet is taken into consideration is also unlikely to be necessary 
based on the same reason described above. This is the first study demonstrating that WGT is a significant covariate in the blood concentration of RFP in Japanese patients, although several studies attempted to elucidate covariates affecting the pharmacokinetics of RFP as described below. Wilkins et al. developed a nonlinear mixedeffects model to describe variable absorption of RFP by using 261 African TB patients aged $18-72$ years and weighing $28.5-85.5 \mathrm{~kg}$ [12]. The model well fitted the observed RFP blood concentration but no variable was determined. In another study performed by Peloquin et al., the population pharmacokinetic data from 23 Caucasian and 1 Hispanic TB patients were analyzed by a nonparametric population method and calculated the covariate by JMPTM software (SAS Institute, North Carolina, U.S.A), but there was no description about the results [13].

Based on the results and discussion described above, this study was able to construct a PPK model required for predicting the pharmacokinetics of RFP in Japanese TB patients. This model is thought to reflect the behavior of RFP in the digestive tract, and by the model one can detect the differences in pharmacokinetics caused by the dietary conditions. Although the relation between the WGT and the exposure is clearly elucidated in the present model because the exposure is a reciprocal of $\mathrm{CL}$, the pharmacokinetics of RFP in children should be examined in a separate trial. In the future, it will be possible to further elucidate the variation factor(s) of pharmacokinetic parameters by further accumulation of related data.

Although guidelines and package inserts of RFP recommend its administration under fasting conditions before breakfast, actually the drug has been pre- and post-prandially given to TB patients in clinical practice in Japan. In this study 31 and 107 patients received RFP before and after meal respectively based on the thought of the concerned physicians; this situation in Japan being largely different from Western countries including EU and USA. The basic goal of current TB medicine is to eradicate TB bacteria living in the patients. The chemotherapy failure is mostly attributable to the interruption of treatment and the incomplete treatment including administration of insufficient amount of RFP in Japan. Our study shows that when the median WGT is $51.5 \mathrm{~kg}, \mathrm{CL}$ is $15.2 \mathrm{~L} / \mathrm{hr}$. When the WGT is $95 \% \mathrm{Cl}$ lower limit $(35.1 \mathrm{~kg}), \mathrm{CL}$ is $10.4 \mathrm{~L} / \mathrm{hr}$, and when the WGT is $95 \% \mathrm{Cl}$ upper limit $(76.5 \mathrm{~kg}), \mathrm{CL}$ is $22.4 \mathrm{~L} / \mathrm{hr}$. Thus, adherence of the RFP dosage per BWG is significantly important. For this reason, it is desirable to provide a guidance or indication stating that TB patients should strictly comply with regular medication and that healthcare professionals must treat the patients appropriately with sufficient effective dosage of RFP based on the scientific evidence.

We have thought that the result of this study reflects the actual clinical circumstances in Japan and is significant in this context.

In Japan, the high morbidity rate of TB remains in comparison with that in developed countries, and is also an issue which we cannot ignore from the viewpoint of the opportunistic infection of HIV. Under these circumstances, the kinetic model of RFP we examined here has been thought to contribute to the improvement of the treatment of TB significantly.

Since the patients involved in this analysis were those whose pharmacokinetics was examined during repeated dosing, and the drug metabolism of RFP may be different between the single and the repeated administration, the results of this study will be limited to the estimation of repeated dose.

\section{Conclusion}

The present analyses demonstrate that the F1, Ka, and zero order absorption time were affected by meal and a WGT selected as the covariate by the SCM highly affected the $C L$ in the range from $-31.6 \%$ to $47.4 \%$. The WGT which was able to be calculated from the power model was selected as covariate affecting $C L$ by the Stepwise Covariate Model method. We concluded that the dose of Japanese patients with tuberculosis can be well estimated by the power model formula.

\section{Acknowledgement}

We express our special thanks to Professor Koichiro Atsuda for giving us careful and kind guidance on the completion of this paper. Also, we would like to express my gratitude to Dr. Satofumi lida, who provided special guidance in carrying out this study.

\section{Sources of Funding}

There was no funding for this study.

Conflicts of Interest

The authors declare that they have no conflict of interest.

\section{References}

[1] 2018 mini-note of TB statistics in Japan. The Tuberculosis Surveillance Center. Available at: https://www.jata.or.jp/rit/ekigaku/en/statisticsof-tb/, Accessed Jan.14, 2020

[2] Statistics of tuberculosis in 2018 (Summary). The Tuberculosis Surveillance Center. Available at: https://www.jata.or.jp/rit/ekigaku/ en/statistics-of-tb/, Accessed Jan.14, 2020

[3] WHO TB burden estimates. https://extranet.who.int/tme/generateCSV.asp?ds = estimates, Accessed Feb 72020

[4] Kekkaku no kijun no kaitei (Revision of the Standards for TB Medicine) 2018 (in Japanese). Kekkaku 2018; 93: 61-68

[5] Prakash J, Velpandian T, Pande JN et al. Serum rifampicin levels in patients with tuberculosis: effect of P-glycoprotein and CYP3A4 blockers on its absorption. Clin Drug Investig 2003; 23: 463-472

[6] Chigutsa E, Visser ME, Swart EC et al. The SLCO1B1 rs4149032 Polymorphism Is Highly Prevalent in South Africans and Is Associated with Reduced Rifampicin Concentrations: Dosing Implications. Antimicrob Agents Chemother 2011; 55: 4122-4127

[7] Panchagnula R, Rungta S, Sancheti P et al. In vitro evaluation of food effect on the bioavailability of rifampicin from antituberculosis fixed dose combination formulations. Farmaco. 2003; 58: 1099-1103

[8] Zent C, Smith P. Study of the effect of concomitant food on the bioavailability of rifampicin, isoniazid and pyrazinamide. Tuber Lung Dis 1995; 76: 109-113

[9] Imai E, Iseki K, Nitta K et al. Clinical Practice Guidebook for Diagnosis and Treatment of Chronic Kidney Disease. Nihon Jinzo Gakkai Shi 2012; 54: 1034-1191 
[10] Kubo H, Kinoshita T, Kobayashi Y et al. Determination of rifampicin in serum by high performance liquid chromatography (Japanese). Bunseki Kagaku 1982; 31: 175-179

[11] Agrawal S, Panchagnula R. Implication of biopharmaceutics and pharmacokinetics of rifampicin in variable bioavailability from solid oral dosage forms. Biopharm Drug Dispos 2005; 26: 321-334
[12] Wilkins J], Savic RM, Karlsson MO et al. Population Pharmacokinetics of Rifampicin in Pulmonary Tuberculosis Patients, Including a Semimechanistic Model to Describe Variable Absorption. Antimicrob Agents Chemother 2008; 52: 2138-2148

[13] Peloquin CA, Jaresko GS, Yong CL et al. Population pharmacokinetic modeling of isoniazid, rifampicin, and pyrazinamide. Antimicrob Agents Chemother 1997; 41: 2670-2679 